\title{
Whole Exome Sequencing Identifies a Novel CoL1A1 Missense Mutation Causing Dentinogenesis Imperfecta Type I Without Skeletal Abnormalities
}

\author{
Yuting Zeng \\ Southern Medical University \\ Yuhua Pan \\ Southern Medical University \\ Jiayao Mo \\ Southern Medical University \\ Zhiting Ling \\ Southern Medical University \\ Lifang Jiang \\ Southern Medical University \\ Fu Xiong \\ Southern Medical University \\ Wenjuan Yan (D645613053@qq.com ) \\ Southern Medical University
}

\section{Research}

Keywords: dentin, odontogenesis, type I collagen, missense mutation, odontoblasts, hardness test

Posted Date: April 22nd, 2021

DOI: https://doi.org/10.21203/rs.3.rs-407777/v1

License: (c) (i) This work is licensed under a Creative Commons Attribution 4.0 International License. Read Full License 


\section{Abstract}

Background冈Osteogenesis imperfecta (OI) is a genetic disorder characterized by bone fragility, blue sclerae and dentinogenesis imperfecta (DGI), which are mainly caused by a mutation of the COL1A1 or COL1A2 genes that encode type I procollagen.

Methods: The ultrastructure of dentin was analyzed by micro-CT, scanning electron microscopy, energydispersive spectroscopy analysis, nanoindentation test and Toluidine Blue Staining. Whole-exome sequencing (WES) was performed to identify the pathogenic gene. The function of the mutant COL1A1 was studied by real-time PCR, western blotting, subcellular localization. Functional analysis in dental pulp stem cells (DPSCs) was also performed to explore the impact of the identified mutation on this phenotype.

Results: WES identified a missense mutation (c.1463G > C) in exon 22 of the COL1A1 gene. However, the cases reported herein only exhibited DGI-I in the clinical phenotype, there is no bone disease and any other common abnormal symptom caused by COL1A1 mutation. In addition, ultrastructural analysis of the tooth affected with non-syndromic DGI-I showed that the abnormal dentin was accompanied by disruption of odontoblast polarization, reduced numbers of odontoblasts, loss of dentinal tubules, and reduction in hardness and elasticity, suggesting severe developmental disturbance. What's more, the odontoblast differentiation ability based on DPSCs that were isolated and cultured from the DGI-I patient was enhanced compared with those from an age-matched, healthy control.

Conclusion: This study helped the family members to understand the disease progression and provided new insights into the phenotype-genotype association in collagen-associated diseases and improve clinical diagnosis of OI/DGI-I.

\section{Introduction}

Dentinogenesis imperfecta (DGI) is a rare autosomal dominant disease that is traditionally classified as DGI-I, DGI-II and DGI-I, which represent a group of hereditary developmental conditions that affect the structure and composition of dentine[1]. While types II and III involve only the teeth, type I is the dental manifestation of osteogenesis imperfecta (OI)-a connective tissue disorder characterized by bone fragility, which may be associated with blue sclerae, DGI and hearing loss. OI is traditionally classified as type I, type II, type III, and type IV, which ranges from very mild types with nearly no fractures through variable skeletal deformities to intrauterine fractures and perinatal death [2, 3]. However, as the high heterogeneity of patients with $\mathrm{Ol}$, the traditional classification could not establish a definitive clinical diagnosis can be difficult, particularly without biochemical or molecular genetic information[4].

Mutations in the type I collagen genes, COL1A1 and COL1A2, have been identified in approximately $90 \%$ of cases with OI. As we all known, OI can damage the life quality of the patients because the main causative gene, type I collagen, is the major structural protein of bone, dentin, and other fibrous tissues[57]. Therefore, we can think that the mutation in type I collagen gene might alter the collagen fibrils, which 
may affect the formation and stability of bone and dentin minerals and finally result in a variety of abnormal phenotypes[8]. Although a lot of type I collagen genes mutations had been reported, DGI without OI has never been linked with $\operatorname{COL} 1 \mathrm{~A} 1$ mutations $[9,10]$, and little is known on phenotype changes of dentin structure and ultrastructure in patients with DGI-I[11-13].

The main pathological feature in DGI-I is the abnormality of dentin mineralization. Mineralization represents a homeostasis and depends on the normal differentiation of human dental pulp stem cells (DPSCs) $[14,15]$. Moreover, DPSCs are highly considered for odontogenesis and reparation of pulp tissue[16]. Interestingly, thus far, no data exist on the potential functional roles that DPSCs may have during dentin development in DGI-I. On this account, human DPSCs can be a valuable model to investigate odontoblastic differentiation impacted by COL1A1 mutation.

Here, we describe a patient was heterozygous for the novel mutation c.1463G>C (p.G488A) in COL1A1. Notably, she didn't have any bone problems or other phenotypes associated with OI, but only clinically evident DGI phenotypes such as opalescent teeth, obliterated pulp chambers and marked cervical constriction of bulbous crown. Meanwhile, we elucidate morphological alterations of defective dentin in patients affected by DGI-I, by ultrastructural and DPSCs-based analyses. In this study, we report that COL1A1 mutation causes non-syndromic human DGI-I.

\section{Materials And Methods}

\section{Patient and clinical examination}

An otherwise healthy 18-year-old Chinese female presented with abnormity of tooth colour, came to Nanfang Hospital (Guangdong province, China) for specific treatment. Clinical assessment and radiographic examinations were performed on the subjects of the family. All procedures in this study were approved by the institutional review board and ethics committee of Nanfang Hospital, an affiliate of Southern Medical University.

\section{Micro-CT Analysis}

With their informed consent, the wisdom teeth extracted from patient (III:2) and the age-matched control female were subjected for ultrastructural analysis. To obtain detailed 3D structural information inside the samples, micro-CT was performed using a $\mu$ CT-Sharp (Micro-M90冈China) with the following settings:70 $\mathrm{kV}, 100 \mu \mathrm{A}$, an isotropic resolution of $20 \mu \mathrm{m}$ and a scan angle of $360^{\circ}$. 3D models of the teeth and dental pulp were reconstructed with Med Project analysis software. The CT images were calibrated using hydroxyapatite mineral of known densities $\left[0.25 \mathrm{~g} \cdot \mathrm{cm}^{-3}\right.$ and $\left.0.75 \mathrm{~g} \cdot \mathrm{cm}^{-3}\right]$ as elsewhere reported[40]. Measurement of the mineral density of the enamel and dentine of each tooth was carried out using Image $\mathrm{J}$ software.

\section{Scanning electron microscopy (SEM)}


The whole tooth sample was embedded in epoxy and sectioned into slices at a thickness of $5 \mathrm{~mm}$ along the mesial-distally plane using a precision cutter. Samples were sputter-coated with gold using an auto sputter coater (Agar Scientific, Elektron Technology, UK). A Hitachi SU-70 scanning electron microscope (Hitachi, Japan) was used to observe the microstructure of samples at $\times 1 \mathrm{k}$ and $\times 10 \mathrm{k}$ magnifications.

\section{Energy-Dispersive Spectroscopy (EDS) analysis}

EDS analysis was realized on the same teeth evaluated for SEM observations. Quantitative element analysis of $\mathrm{Ca}, \mathrm{P}, \mathrm{Na}$ and $\mathrm{Mg}$ was carried out and quantitative analysis to locally determine the composition of the target tissue (in weight \%).

\section{Toluidine Blue Staining}

The teeth without decalcification were mesial-distally cut at a thickness of $10 \mu \mathrm{m}$ with Leica Histocore Autocut (Germany). The slices were stained with $1 \%$ toluidine blue (TB) and observed under Leica DMI6000B (Leica Microsystems, Germany).

\section{Nanoindentation test}

The tooth slices were polished until no discernible scratches could be seen under an optical microscope. The Hardness and Young's modulus of enamel and dentin were measured by using nanoindentation instrument (TI-900, Tribolndenter, Hysitron, USA) with Berkovich diamond indenter. The detecting areas were randomly selected at a distance of $1 \mathrm{~mm}$ along enamel-dentinal junction (EDJ). The experimental parameters are as follows: the strain rate is $0.05 \mathrm{~s}^{-1}$, the depth limit is $2 \mu \mathrm{m}$, peak hold time for $10 \mathrm{~s}$, $200 \mu \mathrm{m}$ apart[41, 42]. The drift rate of the material caused by temperature fluctuations in the environment was monitored to correct all test data throughout a loading-hold-unloading cycle for each indentation test.

\section{Mutation analyses}

Genomic DNA was extracted from peripheral blood of the proband by phenol-chloroform method and was delivered to the Genesky-Shanghai (China) for whole-exome sequencing (WES) analysis. Then, to confirm the causative mutation, co-segregation analyses in all family members were performed. High-resolution melting analyses using 200 genomic DNA samples from random individuals were performed to investigate the mutation frequency in the general population.

\section{Plasmid construction and site-directed mutagenesis}

Full-length human COL1A1 was cloned into the pEGFP-C1 vector, which was cooperated with Hunan Fenghui Biotechnology (China), and COL1A1-Wild Type (WT) was obtained. The c.1463G>C mutant (MUT) was generated using the following primers: forward, 5'-CCCCTGGCGAGCGTGCTGGACCTGGTAGC3'; and reverse, 5'-GCTACCAGGTCCAGCACGCTCGCCAGGGG-3'. Recombinant plasmids were purified using the Plasmid Miniprep Kit (Vazyme, China) and sequenced to exclude random mutants. 


\section{Cell transfection and Subcellular localization}

HEK293 cells were cultured in 12-well dishes and transfected with WT or MUT plasmids using Lipofectamine $^{\mathrm{TM}} 2000$ transfection reagent (Invitrogen). After $36 \mathrm{~h}$ of transfection, the cells were rinsed three times with phosphate-buffered saline (PBS, Sigma-Aldrich, USA)『and nuclei were stained with $0.1 \mu \mathrm{g} / \mathrm{ml}$ 4', 6-diamidino-2-phenylindole (DAPI, Sigma) for $10 \mathrm{~min}$ at room temperature. Subsequently, a confocal fluorescence microscope (LSM 880, Carl Zeiss AG, Germany) was then used to image the cells.

\section{Bioinformatics}

To further confirm the function of mutant COL1A1, the 3D structures of WT and mutant COL1A1 were predicted in silico using I-TASSER (http://zhanglab.ccmb.med.umich.edu/I-TASSER/), and the functional effects of the mutant protein were estimated with Polyphen-2 (http://genetics.bwh.harvard.edu/pph2/).

\section{Quantitative real-time polymerase chain reaction}

Quantitative RT-PCR was applied to examine the expression of COL1A1هDSPP and OCN. After 36 hours transfection of HEK293 cells or after 14 days odontogenic differentiation of hDPSCs, total RNA was isolated using Trizol reagent (Invitrogen) and reverse transcribed into CDNA using the PrimeScript ${ }^{\text {TM }}$ RT reagent Kit (Takara, China). These genes primers have been published elsewhere[43, 44]. Gene expression levels were calculated using the $\left(2^{-\triangle \Delta C T}\right)$ method.

\section{Western blotting analysis}

Western blot was applied to examine the expression of COL1A1هDSPP and OCN. After 36 hours transfection of HEK293 cells or after 14 days odontogenic differentiation of hDPSCs, cells were collected and washed with cold PBS and lysed with cell lysis buffer (Beyotime, China) supplemented with 1\% phenylmethanesulfonylfluoride (PMSF, Beyotime) to prevent protein degradation. Total protein $(20 \mu \mathrm{g})$ was separated by $10 \%$ SDS-polyacrylamide gel and transferred onto a polyvinylidene difluoride (PVDF) membrane (Millipore, USA). After being blocked in $5 \%$ nonfat milk in Tris-buffered saline containing $0.1 \%$ Tween-20 for $1 \mathrm{~h}$ at room temperature, the membranes were then incubated with anti-EGFP (Ray Antibody Biotech, China), anti-DSPP (Santa Cruz, USA), anti-OCN (Abcam, USA) and anti-GAPDH (Sigma, USA) overnight at $4{ }^{\circ} \mathrm{C}$. The next day, the membranes were incubated for $1 \mathrm{~h}$ at $37^{\circ} \mathrm{C}$ with the corresponding secondary antibodies (Proteintech, China), and the immunoreactive proteins were visualized with the ECL Kit (Beyotime, China) according to the manufacturer's instructions.

\section{Cultivation of hDPSCs and Alizarin Red S staining}

Isolation of hDPSCs was performed as described elsewhere[43]. For odontoblastic differentiation experiments, the cells were cultured in an odontogenic medium (OM), consisting of DMEM, $10 \%$ of FBS, $50 \mathrm{mg} / \mathrm{mL}$ ascorbic acid (Sigma, USA), $5 \mathrm{mM} \beta$-glycerophosphate (Sigma, USA), and $10 \mathrm{nM}$ dexamethasone (Sigma, USA). For ARS staining, when hDPSCs were $70 \%$ confluent, the ordinary medium was replaced with the $\mathrm{OM}$ to induce the odontogenic differentiation. After 14 days, the induced cells were fixed 
for 15 min at room temperature in $4 \%$ paraformaldehyde and then stained for 30 min with $2 \%$ ARS (Beyotime, China).

\section{Statistical analyses}

Results are presented as means \pm standard deviation (SD) of at least three independent biological replicates. Biological replicates were analyzed as at least three technical replicates per experimental point. The significance of differences was determined using one-way analysis of variance. The observed differences were considered statistically significant at $p$ values $<0.05$.

\section{Results}

\section{Clinical phenotype}

The teeth of the proband were typically amber and translucent and show significant attrition, especially in molar teeth (Fig. 1a-e). Radio-graphic examination of the teeth revealed bulbous crowns with prominent cervical constrictions. The pulp chambers and root canals of affected teeth were smaller than normal or completely obliterated (Fig. 1f). Radiographs of limb bones and knee revealed no significant osteopenia, bony destructive process, periosteal reactions, or evidence of any acute fractures, dislocations, or injuries (Fig. 1g-j). Besides, bone mineral density, serum calcium, alkaline phosphatase, sclera and echocardiography revealed no remarkable findings. The overall characteristics of the clinical and radiographic results supported a clinical diagnosis of DGI-I (Fig. 4a).

\section{Ultrastructure of the teeth}

Micro-CT analysis shown that a bulbous shape and color change in the proband teeth (Fig. 2a) meanwhile the 3D image of pulp showed an irregularly obliterated pulp chamber and scattered pulp stones. The mineral density measurement showed that the DGI-I teeth had similar scores in the enamel, but lower scores in the dentin compared to the control teeth (Fig. 2b). The SEM images of the control dentin showed the regularly organized dentin tubes and an evenly calcified matrix, while the DGI-I teeth presented very few dentin tubules and enlarged malformed dentin tubes (Fig. 2C). At high magnification, the peritubular dentin of the control teeth is highly calcified and minerals are densely packed, however, the peritubular dentin is more porous and unevenly less calcified. The TB staining observed that there was severe disorganization of the dentin tubules in DGI-I teeth with more irregular dentin in the area towards the pulp. Moreover, the number of odontoblasts adjacent to the mineralized dentin layer was visibly reduced and an obvious difference in odontoblast morphology was observed among them. The roof odontoblasts of the control teeth were columnar in shape, with the nucleus located at the basal end of each odontoblast. However, in the patient's teeth, the odontoblasts became flattened as a result of lost polarity and the odontoblast layer appeared disorganized (Fig. 2e).

\section{Mechanical properties of enamel and dentine}


Nanoindentation test shown the nanoindentation load-displacement curves of the enamel and dentin, which indicated the dentin hardness values and elastic modulus of the DGI-I teeth were significantly reduced compared to the control values. But there was no difference in enamel value (Fig. 3a). Exact values of mechanical properties (average and standard deviation) are summarized in (Fig. 3c). EDS data analysis shown elemental measurements of $\mathrm{P}$ concentration was lower in the DGI-I teeth than the control teeth, whereas $\mathrm{Na}, \mathrm{Mg}$ and $\mathrm{Ca}$ had no differences (Fig. 3b).

\section{The identified DGI-I showed mutation of COL1A1}

WES analysis showed that a novel heterozygous missense variant (c.G1463C, p.G488A) in COL1A1 exon 22 was found to be the cause for DGI-I in the proband of the family. Sanger sequencing shown that this mutation was not identified in any other members of the family (Fig. 4b). Meanwhile, no mutations are detected in the genomic DNA samples from 200 healthy individuals (data not shown). I-TASSER indicated that the COL1A1 c. $1463 \mathrm{G}>\mathrm{C}$ mutation changed the tertiary structure of the protein, causing the changes of portions of the alpha-helix and random coil structure. The Gly488 position is highly conserved in the other known EDA proteins, suggesting that it has an important function in the protein.

\section{Functional analysisafter plasmid transfection}

As shown in Fig. 5c, there was no differences in the subcellular localization of the MUT versus WT protein. In addition, no difference was observed in the levels of mRNA between cells transfected with the MUT plasmid compared with those transfected with WT plasmid. However, western blot analysis revealed that the expression of mutant COL1A1 protein was increased compared with the WT protein (Fig. 5d).

\section{Changes in odontogenic genes and proteins}

Flow cytometric analysis of the surface markers of hDPSCs and the adipogenic and odontoblast differentiation abilities of hDPSCs were shown in the supplementary information. To determine whether the COL1A1 mutation affected hDPSCs differentiation, we analyzed the changes in the levels of odontogenic-specific mRNA and protein markers in induced hDPSCs using qRT-PCR and western blotting, respectively. The expression of COL1A1, DSPP, and OCN in hDPSCs with the COL1A1 mutation was significantly higher than that in control hDPSCs for 14 days after differentiation (Fig. 6a). Moreover, western blotting showed that the protein expression of these genes in DGI-I hDPSCs was significantly upregulated compared with that in the control hDPSCs after odontoblastic differentiation (Fig. 6b). The results proven that the DGII hDPSCs had a higher odontogenic differentiation ability, and ARS staining confirmed it also (Fig. 6c).

\section{Discussion}

As the most abundant tooth matrix protein, type I collagen plays crucial roles in maintaining the integrity of tooth structure and tooth strength. It is an ordered heterotrimer that consisted of two a1 (I) chains and one $\mathrm{a} 2(\mathrm{I})$ chain, which are encoded by COL1A1 and COL1A2 genes, respectively[17]. Mutations in COL1A1 
or COL1A2 show as the following ways: one is quantitative defect including frameshift, nonsense, etc. lead to the synthesis of a reduced amount of normal type I collagen; the other is structural defect including missense mutation, mainly involving glycine replacement within Gly-Xaa-Yaa repeat. In the collagen triple helix, the Gly-substitution missense will produce structural deformation of the triple helix, leading to destabilization of the helical structure, affecting the synthesis of collagen[17-20]. In our study, the singlebase substitution in a Gly codon leading to Ala substitutions, c.1463G>C (p.G488A) (Fig. 4b). Actually, the identity of the residue replacing Gly appears to be closely related to the degree of clinical severity of Ol cases. Substitutions of Gly by Ala, the smallest replacement residue, as we found in the proband only showing DGI-I phenotype, are often mild[21, 22]. Our tertiary structural analysis revealed that the effects of the Gly-substitution in the sequence on the conformation were relatively local (Fig. 5b).

The differences of dentin formation between molars can be related to hard tissue formation development of tooth germs, eruption times and length of exposure to oral factors[23-26]. There was differed greatly phenotype among the molar teeth of the proband. In the proband, the crown of the first molars and second molars displayed totally obliterated pulp chambers, but the third molar teeth only had some irregular pulp stones without excessive dentin formation and obliteration of the pulp cavity, which provide possibility for verifying the odontoblast differentiation ability of dental pulp stem cells in follow-up studies.

Human DPSCs can differentiate into odontoblasts that secrete a mineralized matrix with the mineral and molecular characteristics of dentin, and their normal differentiation is essential for dentin development and formation, which provide a valuable model to investigate odontoblastic differentiation[14]. In this study, we compared the odontogenic abilities of hDPSCs from the proband with a healthy control. For this purpose, we performed ARS staining to monitor mineralization, and we examined the expression levels of the odontogenic differentiation markers DSPP and OCN. The results provided further evidence that the hDPSCs from the mutant proband shown an over-mineralization trend compared with the control and therefore may influence the quality of dentin formation. In addition, the expression of mutant COL1A1 protein was also increased in hDPSCs, which is consistent with the results in HEK293T cells. To the best of our knowledge, the present study is the first to explore the influence of COL1A1 mutation on odontoblastic differentiation based on hDPSCs.

From the view of microstructure, dentin consists of a mineral-rich (or hypermineralized) tubular phase, termed peritubular dentin, next to a collagen-rich fibrillar network phase called intertubular dentin[27]. Then, among fibrillar collagen there contains $85 \%$ type I collagen, $15 \%$ types III and V collagen[28]. Consistent with the former reports, the SEM images of tubules in the mutant dentin are almost completely occluded by peritubular dentin(Fig. 2c), which reduces the apparent size and numbers of the pores[29]. Actually, previous studies have shown increased mineralization to be a characteristic feature of OI bone achieved by densely packed mineral particles as a result of defective collagen, leading to high fragility $[30,31]$. Recently, some scholars proposed that the enlarged dentin collagen fibrils might cause the poorly packed collagen molecules, and finally affect the dentin mineralization[28]. However, we have also observed the quality of mineralization in the DGI-I dentin was far from satisfactory (Fig. 2b). In case of 
the DGI-I, the hardness was found to be significantly lower and the exposed collagen presented overall a lower elasticity than the control samples $(\mathrm{p}<0.05)$, which was consistent with the clinical high brittleness phenomenon (Fig. 3c). In this study the hardness values of normal dentin were in good agreement with the previous studies of dentin[32,33]. And in our studies, the element P in DGI-dentine shown a lower level compared with normal dentine, which verify the positive association between dentin hardness and mineral content $[34,35]$.

Odontoblasts are neural crest-derived cells secreting predentin and dentin and their dysfunctional status may account for a variety of structural changes in dentin from patients with DGI-I[36, 37]. As we seen in the studies, irregular shapes and inverted polarity of odontoblasts further confirmed that the COL1A1 mutation can result in the abnormal dentin. In view of the over-mineralization trend of cultured hDPSCs, abnormal odontoblasts morphology, the decrease hardness of dentin, and the clinical obliterated dental

pulp, we can assume that the initial, slow "entombing" of the dysfunctional odontoblasts is then followed by a fast, disordered matrix deposition and mineralization, eventually leading to complete pulp obliteration[38, 39].

\section{Conclusion}

In conclusion, we report a novel mutation in exon 22 of COL1A1, causing non-syndromic DGI-I in a Chinese family, which expanded the known pathogenic spectrum of COL1A1 gene. And the detailed molecular and clinical features will be useful for exploring phenotype-genotype correlations.

\section{Abbreviations}

OI: Osteogenesis imperfecta; DGI: dentinogenesis imperfecta; WES: Whole-exome sequencing; DPSCs: dental pulp stem cells; SEM: scanning electron microscopy; EDS: energy-dispersive spectroscopy; TB: toluidine blue; EDJ: enamel-dentinal junction; WT: wild type; MUT: mutant type; DMEM: Dulbecco's modified Eagle's medium; FBS: Fetal bovine serum; ARS囚Alizarin Red S staining.

\section{Declarations}

\section{Acknowledgements}

Not applicable.

\section{Authors' contributions}

Yuting Zeng and Yuhua Pan, contributed to design, data acquisition, and analysis, drafted and critically revised the manuscript; Jiayao Mo, Zhiting Ling, Lifang Jiang contributed to data acquisition and analysis, critically revised the manuscript; Fu Xiong and Wenjuan Yan, contributed to conception, design, data analysis, and interpretation, critically revised the manuscript. All authors gave final approval and agree to be accountable for all aspects of the work. 


\section{Funding}

This work was supported by Supported by Natural Science Foundation of Guangdong Province of China (2018A0303130213, 2018B030311033) and Clinical Research Startup Program of Southern Medical University by High-level University Construction Funding of Guangdong Provincial Department of Education (LC2016PY023), as well as Clinical Research Program of Nanfang Hospital Southern Medical University (2018CR018), the President Funding of Nanfang Hospital, China (2019Z018) and the National Natural Science Foundation of China (31970558).

\section{Conflict of interests}

No potential conflict of interest was reported by the authors.

\section{Availability of data and materials}

All data generated or analysed during this study are included in this published article.

\section{Ethics approval and consent to participate}

This study was approved by the Ethics Committee of Nanfang Hospital, Southern Medical University. Written informed consent was obtained from all adult patients.

\section{Consent for publication}

Not applicable.

\section{Competing interests}

The authors have declared no competing interests.

\section{References}

1. Turkkahraman H, Galindo F, Tulu US, Helms JA. A novel hypothesis based on clinical, radiological, and histological data to explain the dentinogenesis imperfecta type II phenotype. Connect Tissue Res. 2020;61:526-36.

2. Zhai N, Lu Y, Wang Y, et al. Splice receptor-site mutation c.697-2A > G of the COL1A1 gene in a Chinese family with osteogenesis imperfecta. Intractable Rare Dis Res. 2019;8:150-3.

3. Kantaputra PN, Sirirungruangsarn Y, Intachai W, et al. Osteogenesis imperfecta with ectopic mineralizations in dentin and cementum and a COL1A2 mutation. J Hum Genet. 2018;63:811-20.

4. Li LJ, Lyu F, Song YW, et al. Genotype-phenotype relationship in a large cohort of osteogenesis imperfecta patients with COL1A1 mutations revealed by a new scoring system. Chin Med J (Engl). 2019;132:145-53. 
5. van Dijk FS, Huizer M, Kariminejad A, et al. Complete COL1A1 allele deletions in osteogenesis imperfecta. Genet Med. 2010;12:736-41.

6. Wang M, Guo Y, Rong P, et al. COL1A2 p.Gly1066Val variant identified in a Han Chinese family with osteogenesis imperfecta type I. Mol Genet Genomic Med. 2019;7:e619.

7. Li R, Wang J, Wang L, et al. Two novel mutations of COL1A1 in fetal genetic skeletal dysplasia of Chinese. Mol Genet Genomic Med. 2020;8:e1105.

8. Marom R, Rabenhorst BM, Morello R. Osteogenesis imperfecta: an update on clinical features and therapies. Eur J Endocrinol. 2020;183:R95-106.

9. Zhang $\mathrm{H}$, Yue $\mathrm{H}$, Wang $\mathrm{C}$, et al. Clinical characteristics and the identification of novel mutations of COL1A1 and COL1A2 in 61 Chinese patients with osteogenesis imperfecta. Mol Med Rep. 2016;14:4918-26.

10. Liu Y, Asan, Ma D, et al. Gene mutation spectrum and genotype-phenotype correlation in a cohort of Chinese osteogenesis imperfecta patients revealed by targeted next generation sequencing. Osteoporos Int. 2017;28:2985-95.

11. Orsini G, Majorana A, Mazzoni A, et al. Immunocytochemical detection of dentin matrix proteins in primary teeth from patients with dentinogenesis imperfecta associated with osteogenesis imperfecta. Eur J Histochem. 2014;58:2405.

12. Eimar H, Tamimi F, Retrouvey JM, et al. Craniofacial and Dental Defects in the Col1a1 Jrt/+ Mouse Model of Osteogenesis Imperfecta. J Dent Res. 2016;95:761-8.

13. Lignon G, Beres F, Quentric M, et al. FAM20A Gene Mutation: Amelogenesis or Ectopic Mineralization? Front Physiol. 2017;8:267.

14. Gronthos S, Mankani M, Brahim J, et al. Postnatal human dental pulp stem cells (DPSCs) in vitro and in vivo. Proc Natl Acad Sci U S A. 2000;97:13625-30.

15. Xin T, Zhang T, Li Q, et al. A novel mutation of MSX1 in oligodontia inhibits odontogenesis of dental pulp stem cells via the ERK pathway. Stem Cell Res Ther. 2018;9:221.

16. Alongi DJ, Yamaza T, Song Y, et al. Stem/progenitor cells from inflamed human dental pulp retain tissue regeneration potential. Regen Med. 2010;5:617-31.

17. Brodsky B, Persikov AV. Molecular structure of the collagen triple helix. Adv Protein Chem. 2005;70:301-39.

18. Rauch F, Lalic L, Roughley P, Glorieux FH. Relationship between genotype and skeletal phenotype in children and adolescents with osteogenesis imperfecta. J Bone Miner Res. 2010;25:1367-74.

19. Shi J, Ren M, Jia J, et al. Genotype-Phenotype Association Analysis Reveals New Pathogenic Factors for Osteogenesis Imperfecta Disease. Front Pharmacol. 2019;10:1200.

20. Sun X, Chai Y, Wang Q, et al. A Natural Interruption Displays Higher Global Stability and Local Conformational Flexibility than a Similar Gly Mutation Sequence in Collagen Mimic Peptides. Biochemistry. 2015;54:6106-13. 
21. Persikov AV, Pillitteri RJ, Amin $P$, et al. Stability related bias in residues replacing glycines within the collagen triple helix (Gly-Xaa-Yaa) in inherited connective tissue disorders. Hum Mutat. 2004;24:3307.

22. Qiu Y, Mekkat A, Yu H, et al. Collagen Gly missense mutations: Effect of residue identity on collagen structure and integrin binding. J Struct Biol. 2018;203:255-62.

23. Brook AH. Multilevel complex interactions between genetic, epigenetic and environmental factors in the aetiology of anomalies of dental development. Arch Oral Biol. 2009;54(Suppl 1):3-17.

24. Dean C, Zanolli C, Le Cabec A, et al. Growth and development of the third permanent molar in Paranthropus robustus from Swartkrans, South Africa. Sci Rep. 2020;10:19053.

25. Christopher Dean M. Tooth microstructure tracks the pace of human life-history evolution. Proc Biol Sci. 2006;273:2799-808.

26. Tziafas D. Characterization of Odontoblast-like Cell Phenotype and Reparative Dentin Formation In Vivo: A Comprehensive Literature Review. J Endod. 2019;45:241-9.

27. Ziskind D, Hasday M, Cohen SR, Wagner HD. Young's modulus of peritubular and intertubular human dentin by nano-indentation tests. J Struct Biol. 2011;174:23-30.

28. Ibrahim S, Strange AP, Aguayo S, et al. Phenotypic Properties of Collagen in Dentinogenesis Imperfecta Associated with Osteogenesis Imperfecta. Int J Nanomedicine. 2019;14:9423-35.

29. Min B, Song JS, Lee JH, et al. Multiple teeth fractures in dentinogenesis imperfecta: a case report. J Clin Pediatr Dent. 2014;38:362-5.

30. Fratzl-Zelman N, Misof BM, Klaushofer K, Roschger P. Bone mass and mineralization in osteogenesis imperfecta. Wien Med Wochenschr. 2015;165:271-7.

31. Boyde A, Travers R, Glorieux FH, Jones SJ. The mineralization density of iliac crest bone from children with osteogenesis imperfecta. Calcif Tissue Int. 1999;64:185-90.

32. Fawzy AS, Priyadarshini BM, Selvan ST, et al. Proanthocyanidins-Loaded Nanoparticles Enhance Dentin Degradation Resistance. J Dent Res. 2017;96:780-9.

33. Yi Q, Feng X, Zhang C, et al. Comparison of dynamic mechanical properties of dentin between deciduous and permanent teeth. Connect Tissue Res. 2020:1-9.

34. Seyedmahmoud R, McGuire JD, Wang Y, et al. The interrelationship of microstructure and hardness of human coronal dentin using reference point indentation technique and micro-Raman spectroscopy. Dent Mater. 2017;33:1069-74.

35. Park H, Hyun HK, Woo KM, Kim JW. Physicochemical properties of dentinogenesis imperfecta with a known DSPP mutation. Arch Oral Biol. 2020;117:104815.

36. Choi SJ, Song IS, Feng JQ, et al. Mutant DLX 3 disrupts odontoblast polarization and dentin formation. Dev Biol. 2010;344:682-92.

37. Hall RK, Maniere MC, Palamara J, Hemmerle J. Odontoblast dysfunction in osteogenesis imperfecta: an LM, SEM, and ultrastructural study. Connect Tissue Res. 2002;43:401-5. 
38. De Coster PJ, Cornelissen M, De Paepe A, et al. Abnormal dentin structure in two novel gene mutations [COL1A1, Arg134Cys] and [ADAMTS2, Trp795-to-ter] causing rare type I collagen disorders. Arch Oral Biol. 2007;52:101-9.

39. Majorana A, Bardellini E, Brunelli PC, et al. Dentinogenesis imperfecta in children with osteogenesis imperfecta: a clinical and ultrastructural study. Int J Paediatr Dent. 2010;20:112-8.

40. Lu T, Li M, Xu X, et al. Whole exome sequencing identifies an AMBN missense mutation causing severe autosomal-dominant amelogenesis imperfecta and dentin disorders. Int J Oral Sci. 2018;10:26.

41. Shen L, Barbosa de Sousa F, Tay N, et al. Deformation behavior of normal human enamel: A study by nanoindentation. J Mech Behav Biomed Mater. 2020;108:103799.

42. Bertassoni LE, Swain MV. Removal of dentin non-collagenous structures results in the unraveling of microfibril bundles in collagen type I. Connect Tissue Res. 2017;58:414-23.

43. Pan $Y$, Lu T, Peng $L$, et al. Vacuolar protein sorting $4 B$ regulates the proliferation and odontoblastic differentiation of human dental pulp stem cells through the Wnt-beta-catenin signalling pathway. Artif Cells Nanomed Biotechnol. 2019;47:2575-84.

44. Zhao Y, Zhai Q, Liu H, et al. TRIM16 Promotes Osteogenic Differentiation of Human Periodontal Ligament Stem Cells by Modulating CHIP-Mediated Degradation of RUNX2. Front Cell Dev Biol. 2020;8:625105.

\section{Figures}



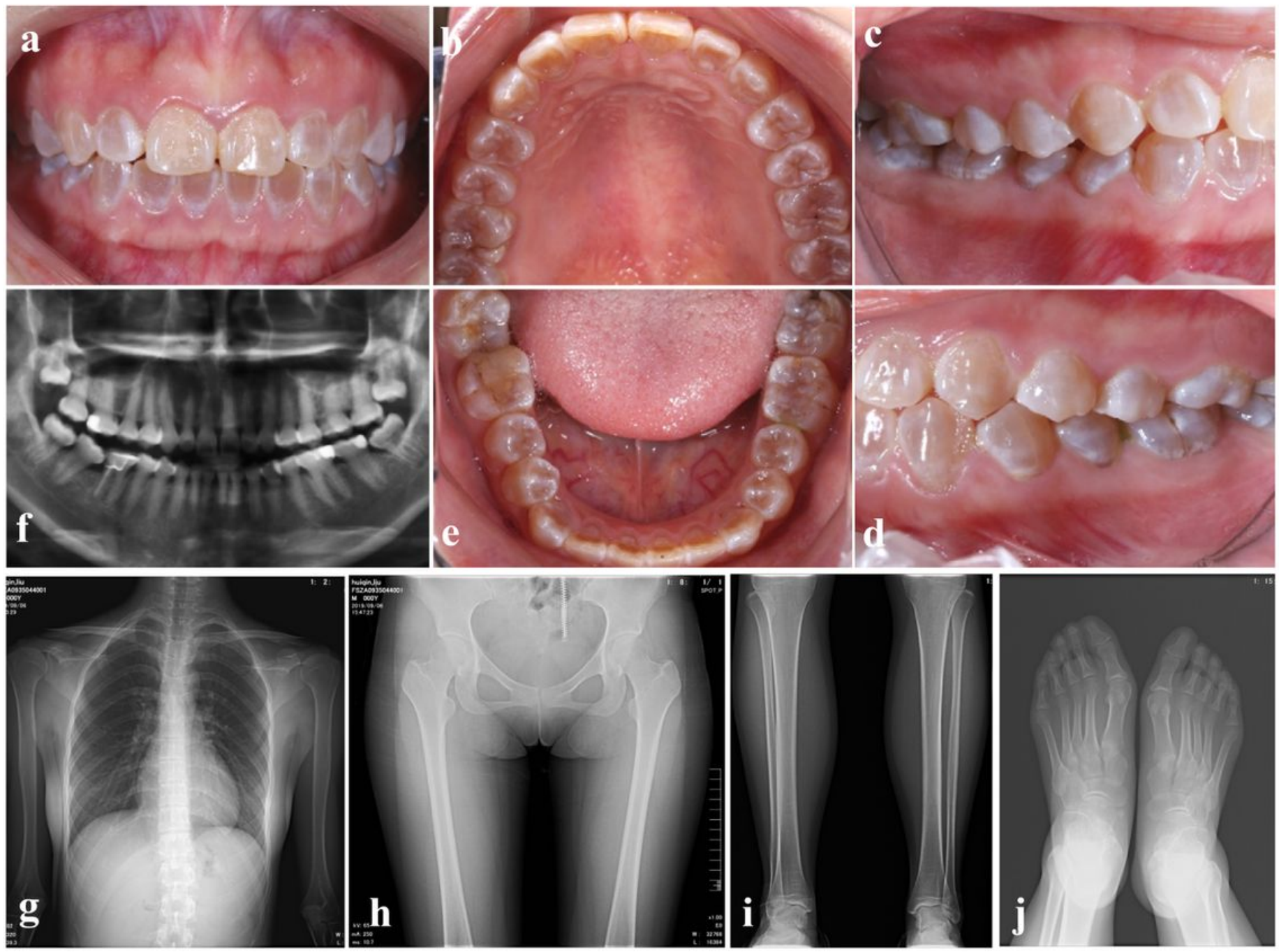

\section{Figure 1}

Clinical images. a-e Intraoral views of the proband. The teeth of the proband were typically amber and translucent and show significant attrition, especially in molar teeth. $\mathrm{f}$-j Panoramic radiographs and radiovisiography images. The pulp chambers and root canals of affected teeth were smaller than normal or completely obliterated. Radiographs of bones and knee revealed no significant osteopenia, bony destructive process, periosteal reactions, or evidence of any acute fractures, dislocations, or injuries. 
a
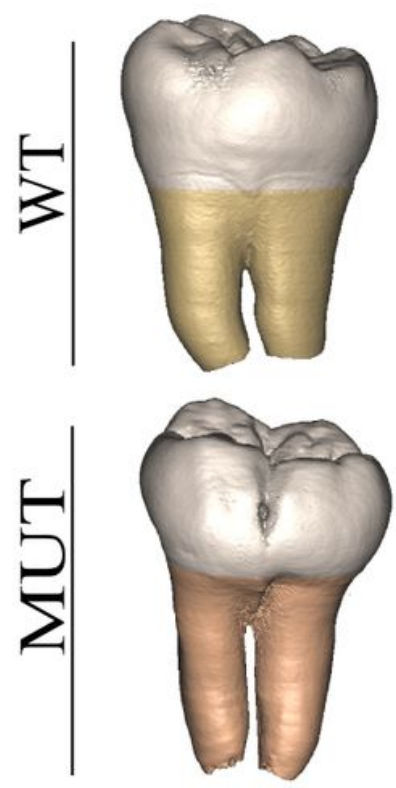

c
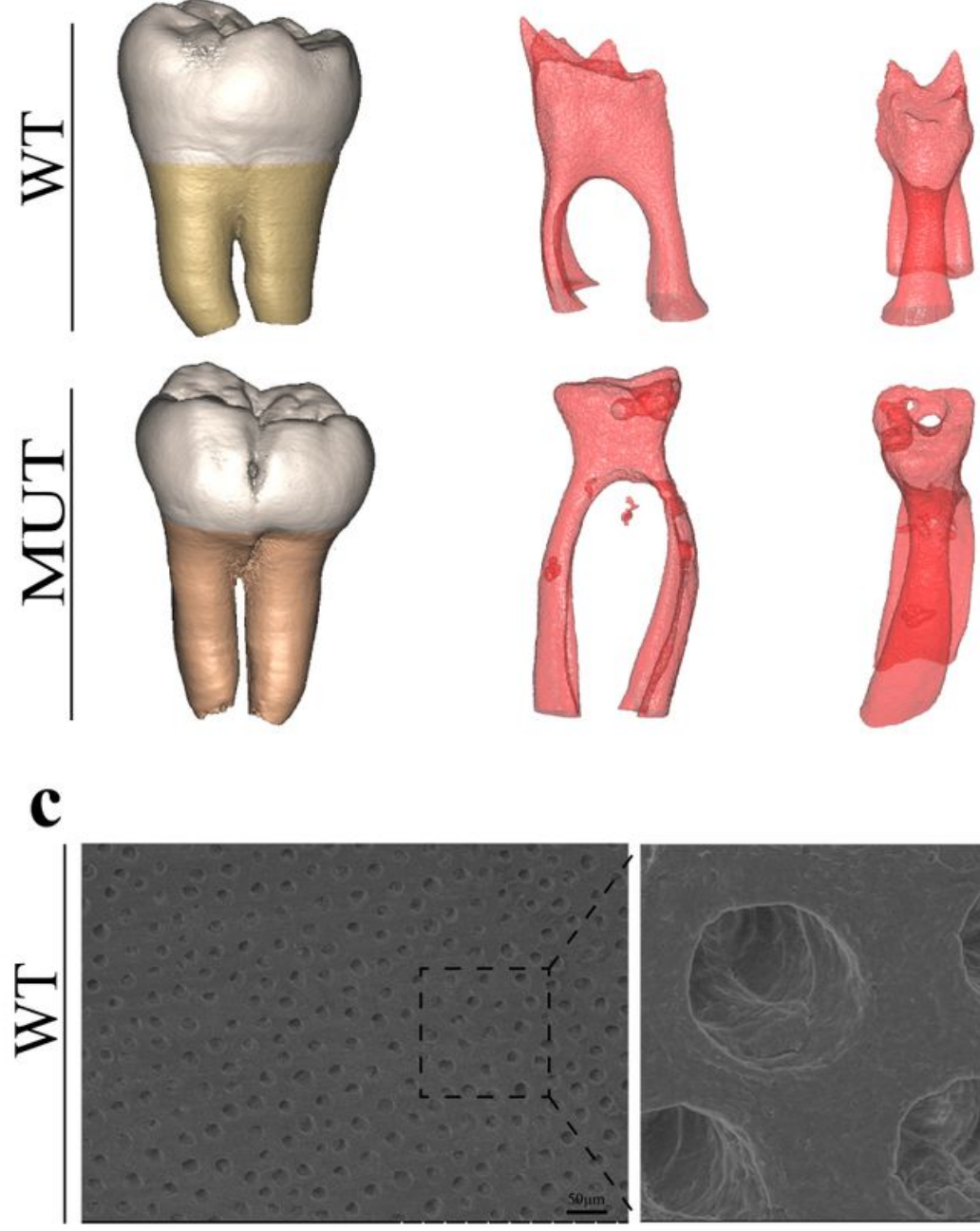

b

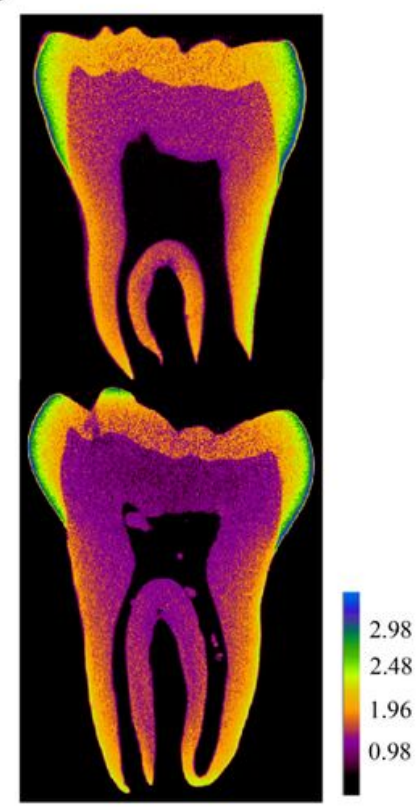

d
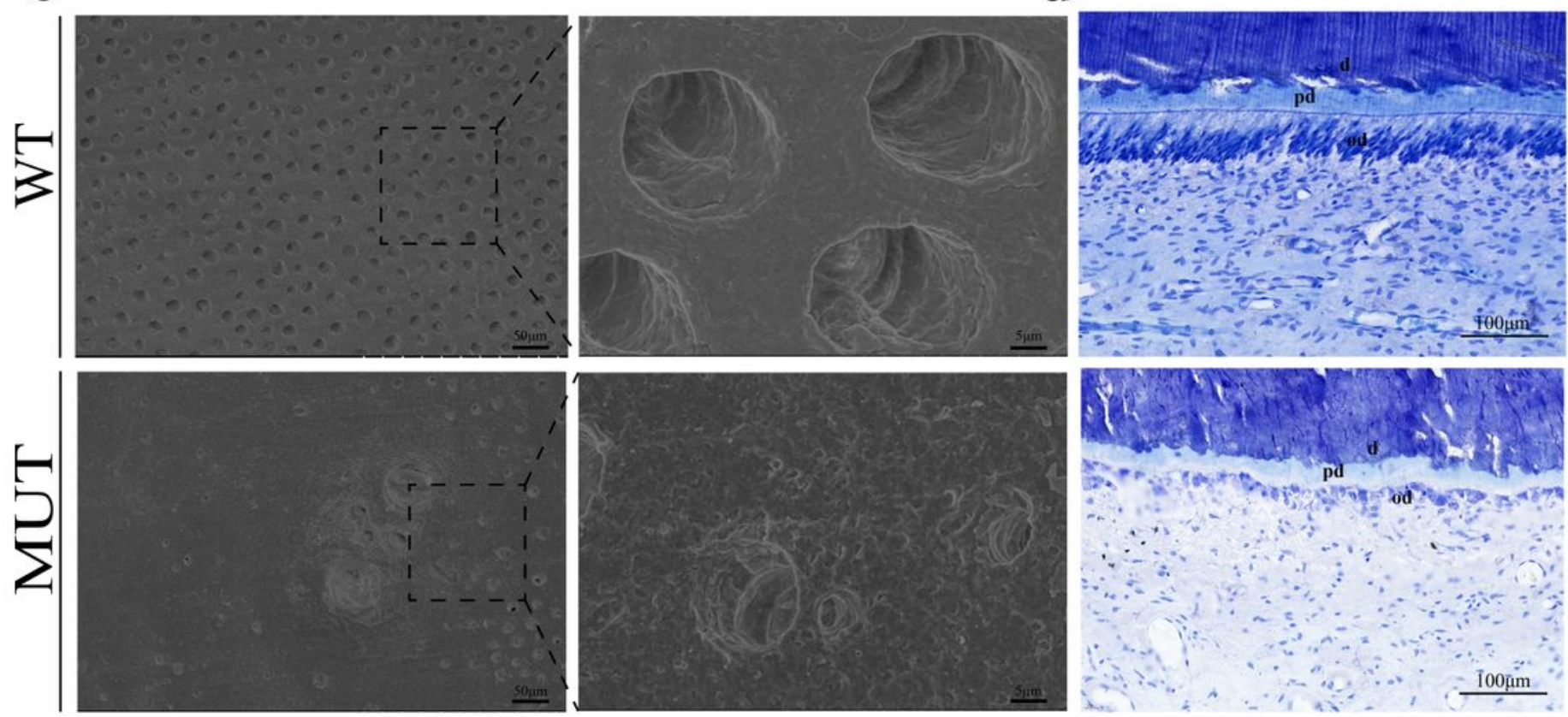

\section{Figure 2}

Teeth ultrastructural analyses. a 3D reconstruction of the tooth CT data. 3D reconstruction of pulp chambers. b Typical CT sections through the teeth are presented using false colour calibrated with respect to mineral density to generate mineral density maps. c SEM of representative exfoliated teeth. The SEM images of the control dentin showed the regularly organized dentin tubes and an evenly calcified matrix, while the DGI-I teeth presented very few dentin tubules and enlarged malformed dentin tubes. $d$ Toluidine blue staining of tooth. The control dentin shows the regularly organized lines, while the proband dentin has irregular lines and waved structures, which are loosely packed. Moreover, the number 
and morphology of odontoblasts adjacent to the mineralized dentin layer were visibly different. $d$, dentin; od, odontoblast; pd, predentin.
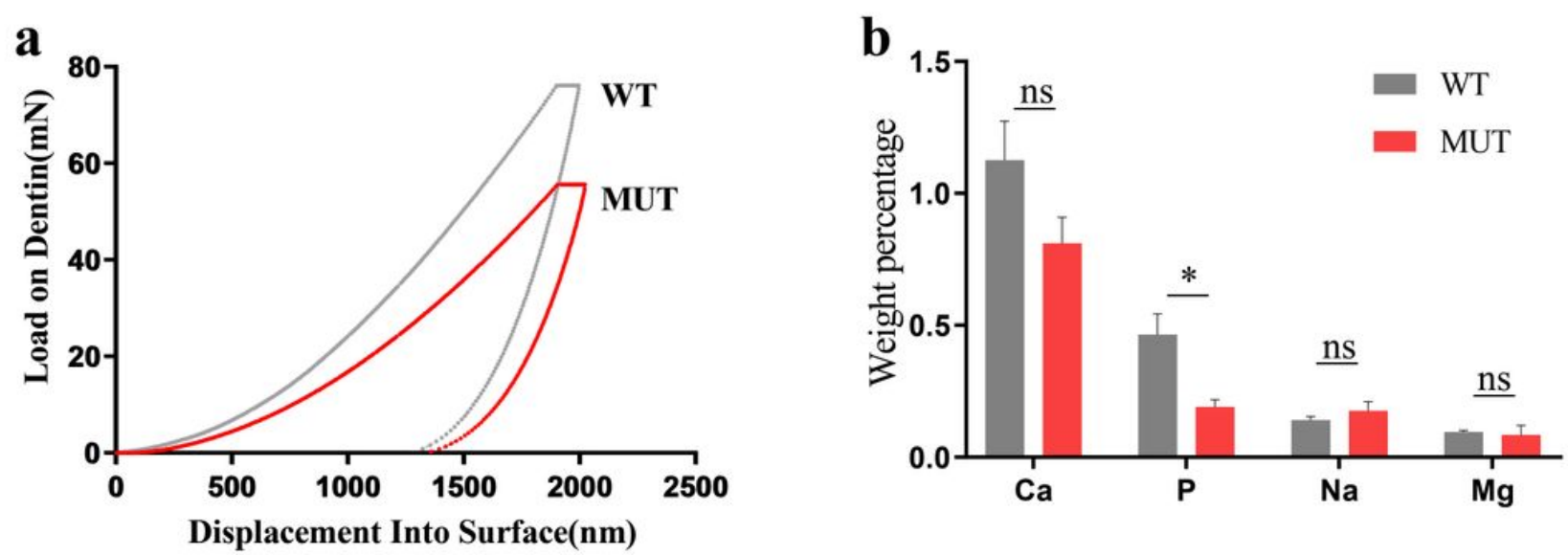

\begin{tabular}{cccc} 
c & & & \\
\hline \multirow{2}{*}{ Group } & Area & Hardness(Gpa) & Modulus(Gpa) \\
\hline \multirow{2}{*}{ WT } & Enamel & $4.32 \pm 0.03$ & $80.92 \pm 3.49$ \\
& Dentin & $0.97 \pm 0.08$ & $22.77 \pm 1.47$ \\
\hline \multirow{2}{*}{ MUT } & Enamel & $4.42 \pm 0.23$ & $79.62 \pm 1.83$ \\
& Dentin & $0.57 \pm 0.09^{* *}$ & $18.07 \pm 1.07$ \\
\hline
\end{tabular}

\section{Figure 3}

a Load-displacement curves for the tooth dentin. b Elemental composition of the normal and affected teeth. Sodium ( $\mathrm{Na})$, magnesium $(\mathrm{Mg})$, phosphorus $(\mathrm{P})$, and calcium $(\mathrm{Ca})$ are shown in separate graphs. c Hardness and modulus on the enamel and dentine of the normal and affected teeth. Values are means \pm SD of three independent experiments $\left({ }^{*} P<0.05\right.$ and $\left.{ }^{*} * P<0.01\right)$. 


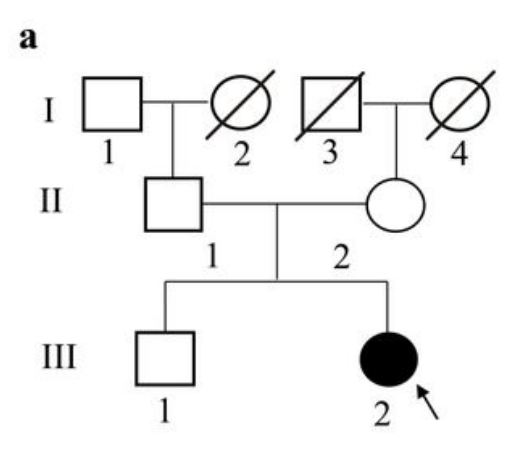

c

\section{COL1A1}

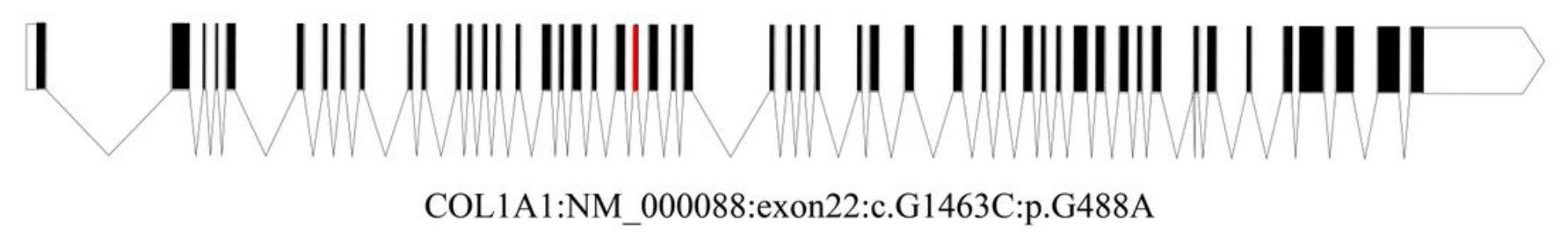

\section{Figure 4}

Mutation analysis in COL1A1. a Pedigree of family. $b$ Sequence chromatogram of affected individual (heterozygous) and control (wild type). c Genomic structure of COL1A1.The rectangles represent exons. 
$\mathbf{a}$

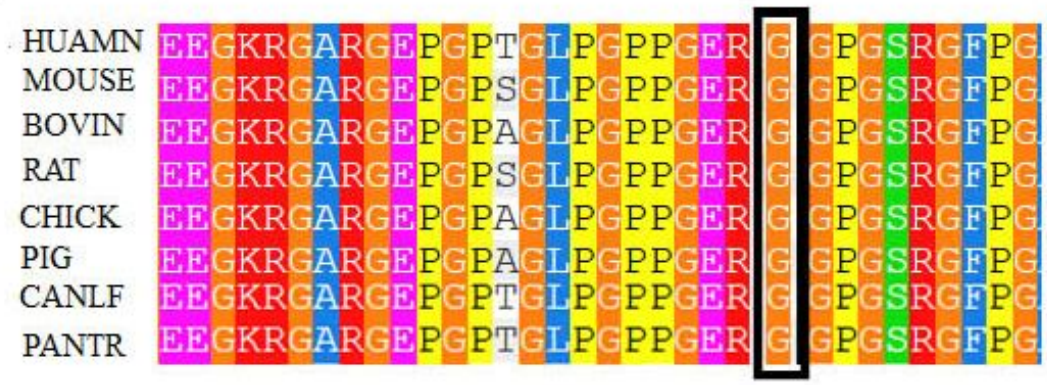

c

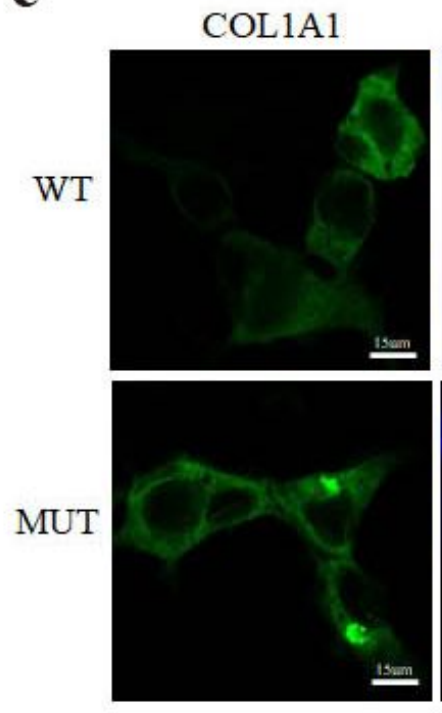

DAPI

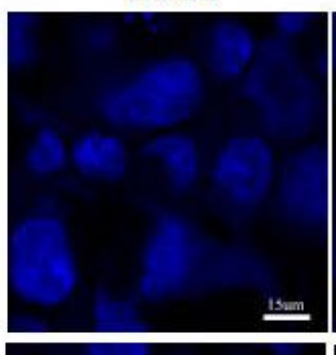

MERGE
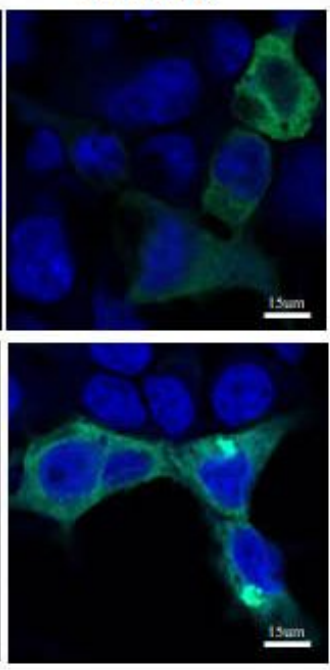

b
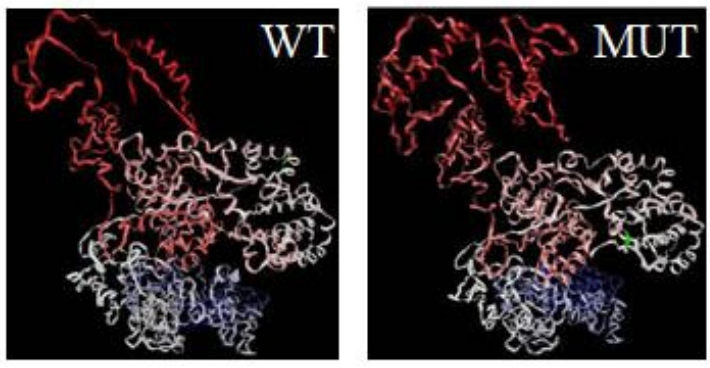

d
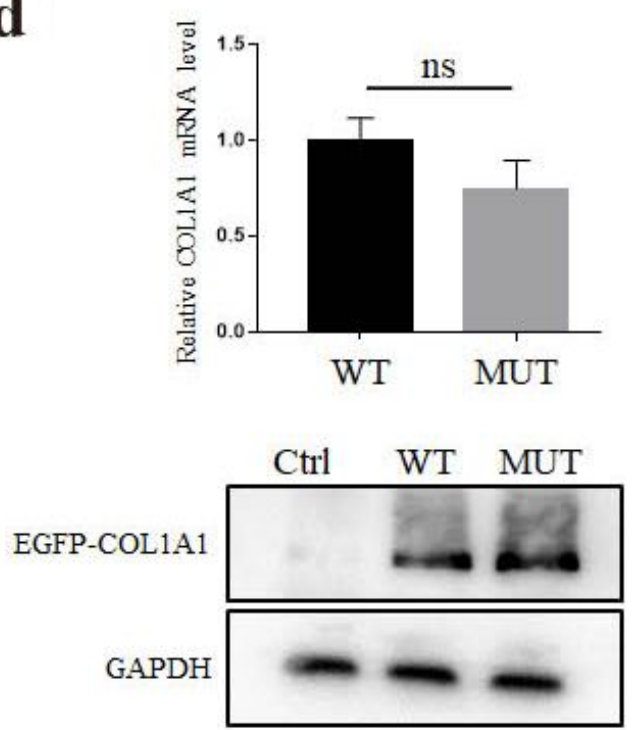

\section{Figure 5}

Effect of mutation on COL1A1 function. a Conservation analysis of this abnormal variation by Polyphen2. The result showed that amino acid 488 COL1A1 was highly conserved between different species. $b$ The 3D structure of mutated COL1A1 was different from that of the wild-type predicted by I-TASSER. C Subcellular localization of COL1A1 in HEK293 cells. The mutant COL1A1 was localized in the cytoplasm similar to the wild-type protein. $d$ The mRNA and protein expression level of COL1A1 in HEK293 cells. Mutant COL1A1 mRNA expression was no different than that of the wild type in HEK293 cells, but the mutant COL1A1 protein expression was increased than that of the wild type $(P>0.05)$. Values are means $\pm S D$ of three independent experiments $\left({ }^{\star} P<0.05\right.$ and $\left.* * P<0.01\right)$ 
a

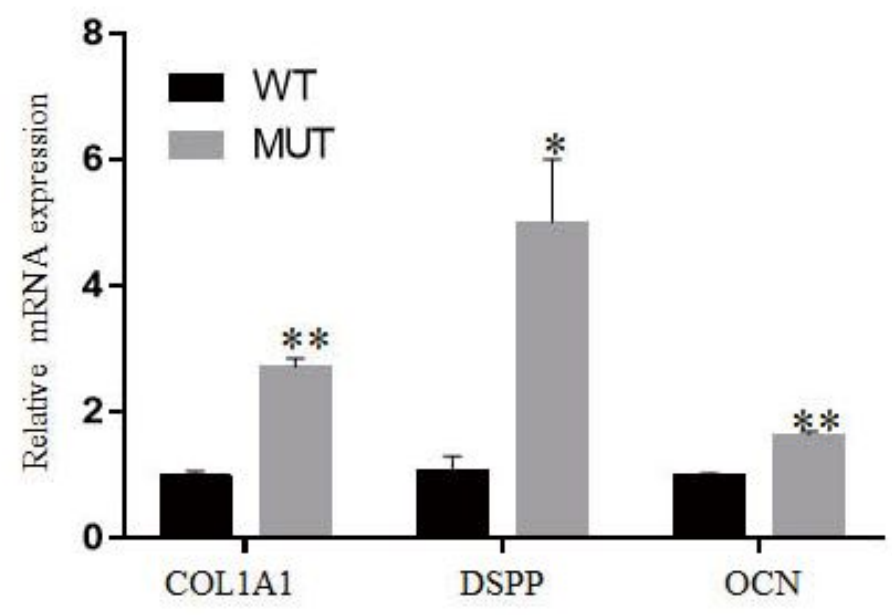

b

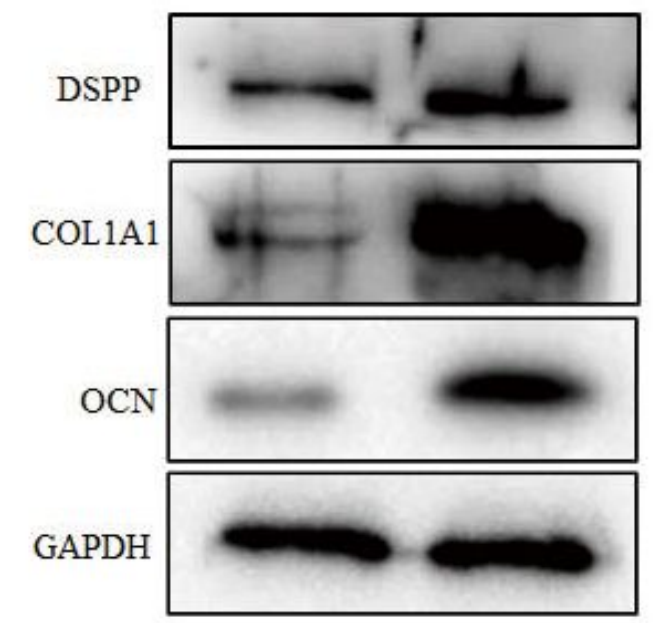

c
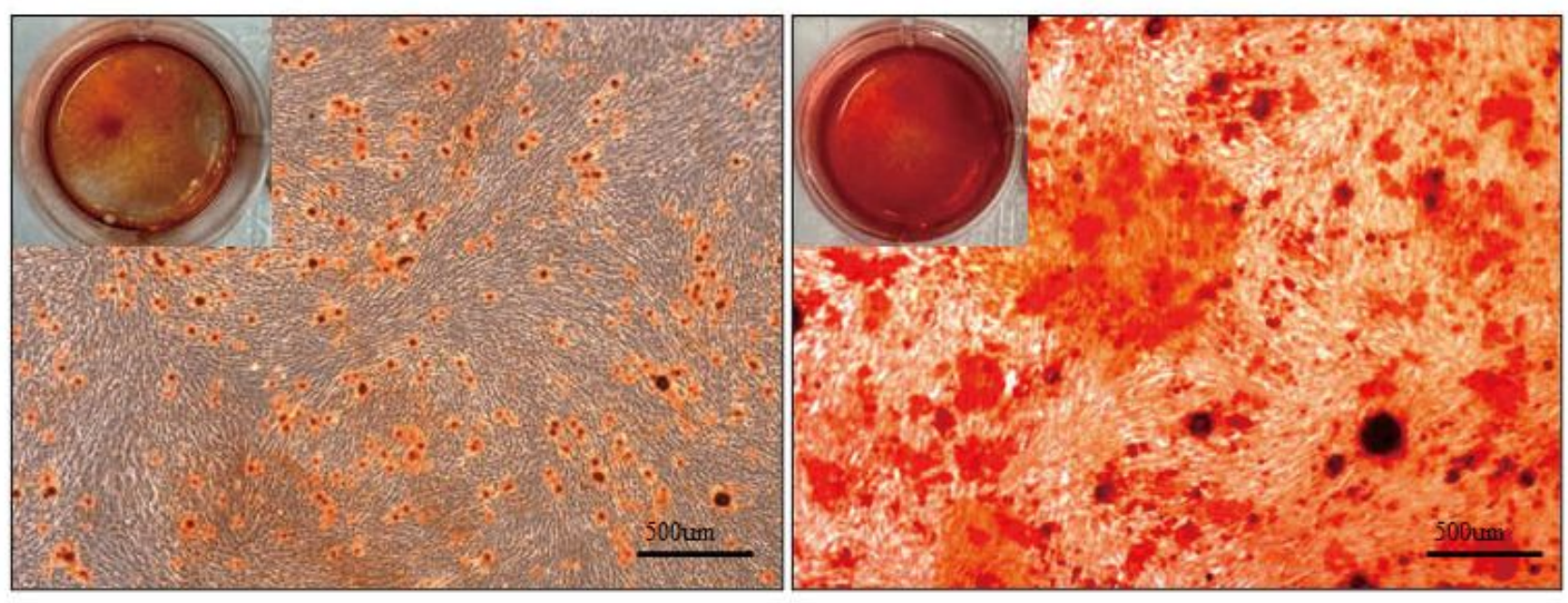

Figure 6

Analysis of the ability of odontogenic differentiation of hDPSCs. a-b Analysis of the mRNA and protein expression of COL1A1 and odontogenic differentiation markers (DSPP and OCN) during the osteogenic differentiation of hDPSCs. Values are means \pm SD of three independent experiments $\left({ }^{*} P<0.05\right.$ and ${ }^{* \star} P<$ 0.01). c Representative images from the ARS staining of DPSCs-MUT and DPSCs-CON at the indicated time points after the induction of differentiation, respectively

\section{Supplementary Files}

This is a list of supplementary files associated with this preprint. Click to download.

- supplementaryFile.doc 